Journal of Molecular Structure (Theochem), 138 (1986) 59-68

Elsevier Science Publishers B.V., Amsterdam - Printed in The Netherlands

\title{
THEORETICAL STUDY OF THE BRIDGING IN $\beta$-HALO ETHYL
}

\author{
BERND ENGELS and SIGRID D. PEYERIMHOFF \\ Lehrstuhl für Theoretische Chemie, Universität Bonn, Wegelerstraße 12, D-5300 \\ Bonn 1 (West Germany)
}

(Received 27 June 1985)

\begin{abstract}
Large-scale multi-reference configuration interaction (MRD-CI) calculations in a quite flexible $\mathrm{AO}$ basis are employed to study the energy hypersurface for the reaction intermediates $\mathrm{XC}_{2} \mathrm{H}_{4}$ with $\mathrm{X}=\mathrm{Cl}, \mathrm{Br}$ and $\mathrm{F}$. Particular emphasis is therby placed on determining the equilibrium conformations, the $\mathrm{CH}_{2}$ rotation barrier and the energy surface for a possible bridging (shuttling motion [1a] of $\mathrm{X}$ between the two carbon centers). The absolute minimum in the potential energy surface is found in all three cases for the asymmetric $\beta$-halo radical in general agreement with ESR data at an XCC angle of ca. $110^{\circ}$, a C-C separation somewhat shorter than a single bond and an approximate $s p^{3}$ type hybridization $\left(\alpha_{2} \cong 135-140^{\circ}\right)$. In $\mathrm{FC}_{2} \mathrm{H}_{4}$ the energy difference between the minimum in the symmetric conformation and the absolute minimum is found to be more than $30 \mathrm{kcal}$ so that shuttling seems impossible in agreement with experimental findings. In $\mathrm{BrC}_{2} \mathrm{H}_{4}$ the difference between these two potential minima is only between $1-2 \mathrm{kcal}$, i.e., smaller than the barrier to $\mathrm{CH}_{2}$ rotation, so that shuttling is favored, while $\mathrm{ClC}_{2} \mathrm{H}_{4}$ takes an intermediate position between these extremes. The use of correlated wavefunctions is found to be quite important for such a study; the results are related to various kinetic studies of these radicals.
\end{abstract}

\section{INTRODUCTION}

In 1962 Thaler [1b] reported the unexpected preponderance of 1,2dibromobutane in the product mixture from the radical bromination of 1-bromobutane and attributed this result to a rate-enhancing effect of the bromo substituent on the vicinal position. Since then many studies have been undertaken [2] in similar radical substitution reactions. They show a smaller effect if bromine is replaced by chlorine, but radical reactions involving fluorine behave in an entirely normal fashion.

Skell and Traynham [3] interpreted the unexpected behavior of the bromine and chlorine compounds by invoking a non-classical bridged radical. For this bridged radical they discussed two possible structures: a symmetrically bridged radical (Fig. 1) with a single equilibrium position, and an unsymmetrical bridged structure (Fig. 2) with a fast shuttling motion of the bridging atom between the two carbon centers. In contrast to a classical (unbridged) radical, both bridged structures allow only restricted rotation about the $\mathrm{C}-\mathrm{C}$ bond, and the halogen atom has control of the stereochemistry 
<smiles>CC12CC(C)(C(I)C1I)C2(C)C</smiles>

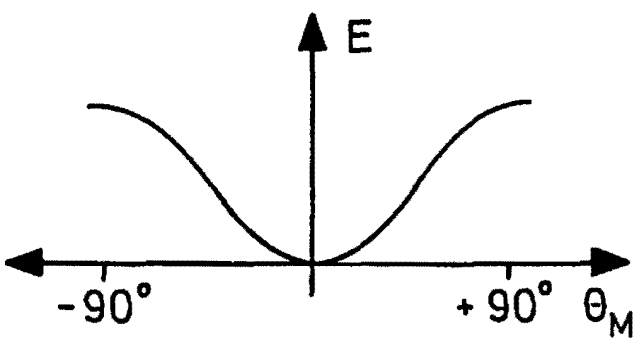

Fig. 1. Schematic representation of a symmetrically bridged radical and its corresponding potential energy surface for the shuttling motion.
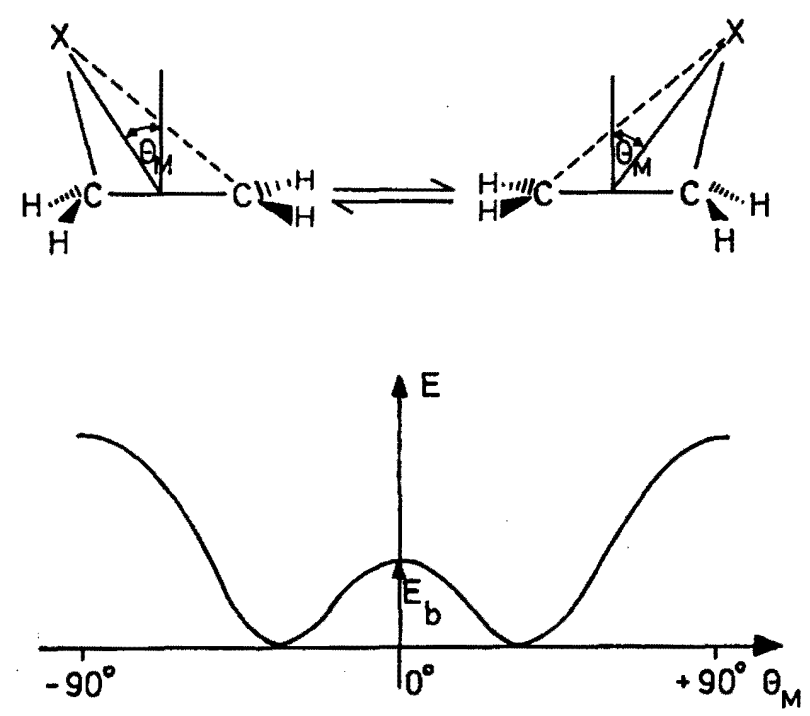

Fig. 2. Schematic representation of an unsymmetrically bridged radical with its corresponding potential energy surface tor the shuttling motion. 
at both carbon centers. This interpretation of experimental results is not free from dispute, however, and alternative theories have been suggested [4] .

Electron spin resonance (ESR) spectra have so far been the most direct approach for studying the structure of such radicals $[5,6]$. It has been found for example, that for a series of alkyl radicals substituted in the $\beta$ position with sulfur, silicon, germanium and tin [5], the isotropic coupling constants for the $\beta$ protons are unusually small and show marked temperature dependence. This result has been interpreted in terms of hindered internal rotation about the $\mathrm{C}_{\alpha}-\mathrm{C}_{\beta}$ bond and a conformational distortion, and on this basis a symmetrically bridged radical has been ruled out. Semiempirical INDO calculations [6] have also been used to study the relation between $\beta$-splitting and conformational preferences.

Since computational quantum chemistry is able to determine entire energy surfaces, it is the goal of this contribution to present potential energy surfaces obtained by configuration interaction methods for the systems $\mathrm{FC}_{2} \mathrm{H}_{4}, \mathrm{ClC}_{2} \mathrm{H}_{4}$ and $\mathrm{BrC}_{2} \mathrm{H}_{4}$; the region of particular interest is thereby the energy minimum of the radical (i.e., its equilibrium structure) and the area in which a possible shuttling motion would take place. This information should contribute to a further understanding of the reaction mechanism. The surfaces for the fluorine and bromine compound are fairly complete, and the work on $\mathrm{ClC}_{2} \mathrm{H}_{4}$ will be finished shortly. A discussion of the detailed results will appear at a later stage [7].

\section{DETAILS OF THE CALCULATIONS}

Preliminary calculations have been carried out employing a $(9 s 5 p)$ set of gaussians given by Huzinaga for the carbon atoms in the [4s2p] contraction suggested by Dunning [8a]. For hydrogen, the five-component expansion by Whitten [9] was used in the [2s] contraction with a scaling factor of $\eta^{2}=2.0$. For the bromine atom various contractions of the $(13 s 9 p 5 d)$ and (14s11p5d) sets, suggested by Dunning [10], were tested. It was finally decided to take a $[9 s 6 p 2 d]$ basis in the $[4,2,1,1,1,1,1,1,1 / 4,1,1,1,1,1 / 4,1]$ arrangement of the smaller AO set; the atomic SCF energy is thereby

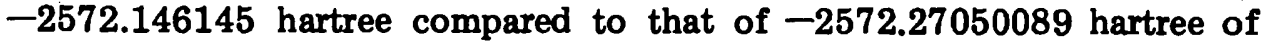
the entirely uncontracted basis. For chlorine the standard $(12 s 8 p)$ basis by Dunning and Hay [8b] in the [6s4p] contraction was used and for fluorine the $(9 s 5 p)$ set in the [4s2p] contraction (5a) was chosen. In order to obtain an adequate description of the $\mathrm{CC}$ bond, an additional $s$-bond function with exponent $\alpha(s)=1.4$ was located between the two carbon atoms. Similarly a bond $s$-function with exponent 1.2 for bromine and chlorine and 1.3 for fluorine was placed in the $\mathrm{CX}$ bond; in most cases it was localized in the center between the halogen and the middle of the CC bond. After the first calculations it soon became obvious that $d$-functions are also required for a good description of polarization and correlation; hence $d$-functions with exponents 0.7 were added to the carbon and fluorine 
set while $\alpha(d)=0.6$ was chosen for chlorine. No additional functions are located at bromine. The total number of contracted gaussians was, therefore: 58 for $\mathrm{FC}_{2} \mathrm{H}_{4}, 66$ for $\mathrm{ClC}_{2} \mathrm{H}_{4}$ and 81 for $\mathrm{BrC}_{2} \mathrm{H}_{4}$.

In all calculations the SCF solution was first determined for the lowest state and the corresponding orbitals were then employed as the basis for the ensuing MRD-CI calculation. Details of the CI work will be presented elsewhere [7]. In $\mathrm{FC}_{2} \mathrm{H}_{4}$ a core of three MOs, corresponding to the $K$-shells of $F$ and $C$ respectively, have always been kept doubly occupied while the three MOs with highest orbital energies have been discarded entirely. Hence the $\mathrm{CI}$ correlates 19 electrons to be distributed among 52 possible orbitals. In $\mathrm{BrC}_{2} \mathrm{H}_{4} 16 \mathrm{MOs}$ corresponding to the $K, L$ and $M$ shells in bromine $\left(1 s^{2} 2 s^{2} 2 p^{6} 3 s^{2} 3 p^{6} 3 d^{10}\right)$ and the $K$-shells of carbon have always been assumed to be doubly occupied while complementary MOs (13 MOs) have not been considered at all. In this way again 19 electrons could be distributed among a total of 52 MOs. Generally two different CI calculations are undertaken, one with only a single and one with a larger number of reference configurations. The total configuration spaces were thus in the order of 500,000 to $>1,000,000$ in $\mathrm{BrC}_{2} \mathrm{H}_{4}$ and of a similar size in $\mathrm{FC}_{2} \mathrm{H}_{4}$. The selection threshold was always $10 \mu \mathrm{h}$ so that the secular equations, when solved had values in the order of 10,000. MRD-CI and estimated full CI energies are evaluated in the standard manner [11].

The coordinate system and the parameters employed in the calculations are shown in Fig. 3. Because of the size of the system, not all parameters have been optimized. The values $\alpha_{1}=180^{\circ}$ and $\beta_{1}=118^{\circ}$ are set to their corresponding values in ethylene. Furthermore all $\mathrm{CH}$-bond lengths are chosen to be equal to $1.086 \AA$. For all optimizing procedures, the angle, $\phi$, is also set to zero and varied only to obtain the $\mathrm{CH}_{2}$ rotational barrier or the corresponding distortion in $\mathrm{FC}_{2} \mathrm{H}_{4}$. All the other parameters $\left(\alpha_{2}, \mathrm{CC}\right.$ and $\beta_{2}$ ) are varied and optimized for each value of $\Theta_{M} R_{M}$, which are the leading coordinates in describing the shuttling motion of the halogen between the two carbons. The surface for $\mathrm{FC}_{2} \mathrm{H}_{4}$ is constructed via a spline

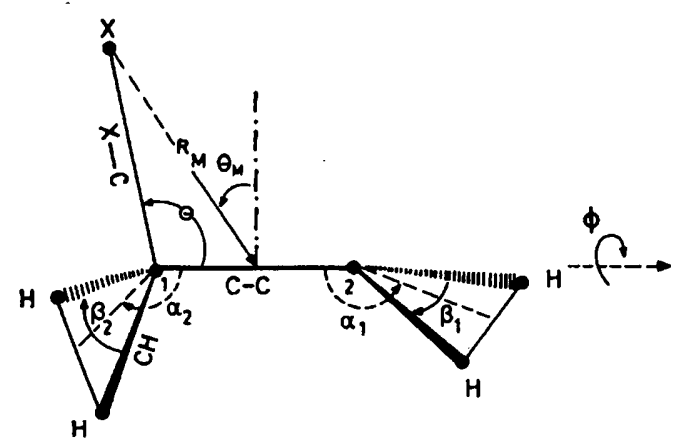

Fig. 3. Coordinate system used in the present calculations. 
fit whilst that for $\mathrm{BrC}_{2} \mathrm{H}_{4}$ is constructed in a somewhat more approximate manner.

\section{THE SYSTEM $\mathrm{FC}_{2} \mathrm{H}_{4}$}

A contour plot of the calculated potential energy for the $\mathrm{FC}_{2} \mathrm{H}_{4}$ surface is shown in Fig. 4. A deep valley is seen for the asymmetric structure which corresponds to the absolute minimum in energy and a flat minimum which corresponds to dissociation is seen at $\Theta_{M}=0$.

The optimal structure calculated by using the MRD-CI treatment has the following parameters: $R_{\mathrm{CC}}=1.50 \AA, \alpha_{2}=140^{\circ}, \Theta_{\mathrm{M}}=41^{\circ}$ and $R_{\mathrm{M}}=$ $1.82 \AA$ which correspond to a molecular angle of $\Theta=108^{\circ}$ and an $\mathrm{F}-\mathrm{C}$ bondlength of $1.44 \AA ; \beta_{2}$ has thereby been held constant at $118^{\circ}$. The total SCF energy for the ground state configuration $1 a^{\prime 2} 2 a^{\prime 2} \ldots 9 a^{\prime 2} 10 a^{\prime} 1 a^{\prime \prime 2} 1 a^{\prime \prime 2} \ldots$ $3 a^{\prime 2}$ is -177.4826 hartree at this point while the MRD-CI energy is -177.873 hartree (for one reference configuration) and the estimated full $\mathrm{CI}$ is -177.907 hartree. The charge of the singly-occupied electron is primarily localized at the terminal carbon $\mathrm{C}_{1}$ (see Fig. 3) but noticeable density is still found at fluorine. The bent skeletal FCC conformation with a bond angle around $110^{\circ}$ is expected on the basis of qualitative MO theory in the Mulliken-Walsh model [12-14] and is typical for molecules with a triatomic skeleton in their electronic ground state and possessing 19 valence electrons. A very similar result was obtained much earlier for the isovalent system $\mathrm{C}_{2} \mathrm{H}_{4} \mathrm{NH}_{2}[15]$.

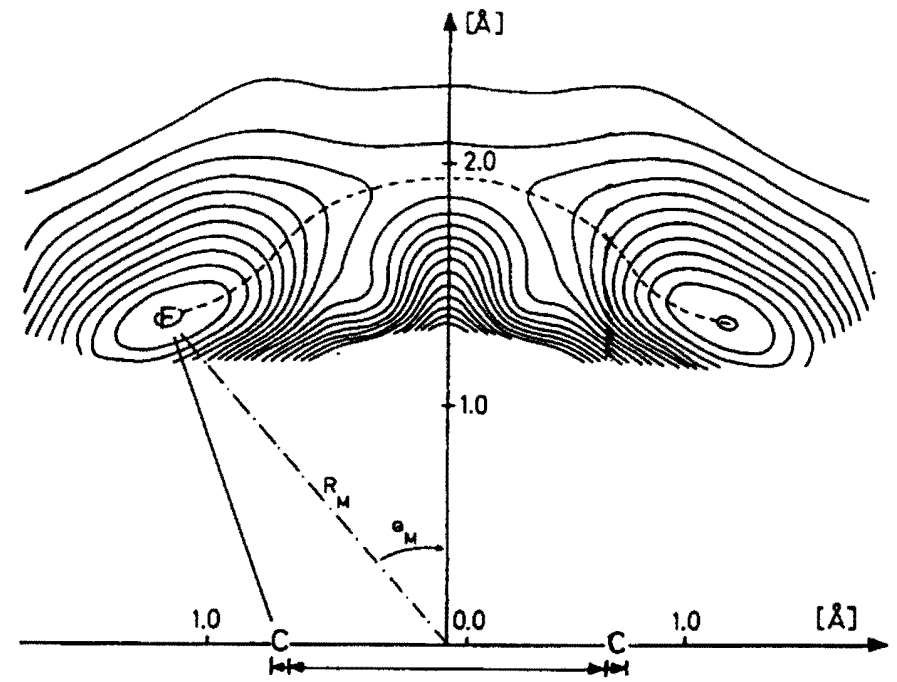

Fig. 4. Calculated contour plot for $\mathrm{FC}_{2} \mathrm{H}_{4}$ in the shuttling region between the two carbon atoms. All parameter are optimized for the $\left(\boldsymbol{R}_{\mathbf{M}}, \boldsymbol{\theta}_{\mathrm{M}}\right)$ pair. The minimum energy path is also indicated. 
A lengthening of the CF bond relative to the optimal structure shows two effects on the other structural parameters: first an increase in the value of $\alpha_{2}$ to a value of $180^{\circ}$, as expected for a separated fluorine atom and ethylene, and secondly a distinct shortening of the $\mathrm{CC}$ bond from the almost single-bond value to that of a double bond. These effects are expected in accordance with the trend from $s p^{3}$ to $s p^{2}$ hydridization upon the removal of the halogen. The angle $\beta_{2}$ is also changed in this process (from $110^{\circ}$ to $118^{\circ}$ ) but this variation is energetically relatively small according to detailed calculations for the $\mathrm{ClC}_{2} \mathrm{H}_{4}$ system. The calculated dissociation energy for $\mathrm{F}-\mathrm{C}_{2} \mathrm{H}_{4}$ is $37 \mathrm{kcal} \mathrm{mol}^{-1}$.

A variation in the parameter $\Phi$ at the present optimized geometry brings about a lowering of approximately $2 \mathrm{kcal}$ in the MRD-CI surface $\left(\Phi \approx 40^{\circ}\right)$ while the effect on the full $\mathrm{CI}$ estimate was below $1 \mathrm{kcal} \mathrm{mol}^{-1}$ (Fig. 5). The change in total energy is quite small over the entire region between $\Phi=0^{\circ}$ and $\Phi=90^{\circ}$ and, therefore, it is difficult to determine unambiguously an optimum value of $\Phi$. The important information to be drawn from this figure is that the minimum in energy is neither at $\Phi=0^{\circ}$ nor at $\Phi=90^{\circ}$, that rotation is quite easy and that the barrier for a torsional vibration towards $\Phi=0^{\circ}$ is smaller than towards $\Phi=90^{\circ}$. It is conceivable that the torsional motion can be confined at lower temperatures to an average angle around $\Phi=0^{\circ}$. Thus the calculations agree quite well with the model given by Edge and Kochi [16] on the basis of their ESR measurements.

Finally, we want to consider the potential surface for a possible shuttling mechanism (Fig. 4). The lowest point for the symmetrically bridged structure is found for $\mathrm{CC}=1.38 \AA, \alpha_{2}=180^{\circ}$ and $R_{\mathrm{M}}=1.9 \AA$. The difference between the absolute minimum at $\Theta_{M}=41^{\circ}$ and this lowest point at $\Theta_{M}=$ $0^{\circ}$ is ca. $33 \mathrm{kcal} \mathrm{mol}^{-1}$. The minimum energy path (MEP) for the shuttling
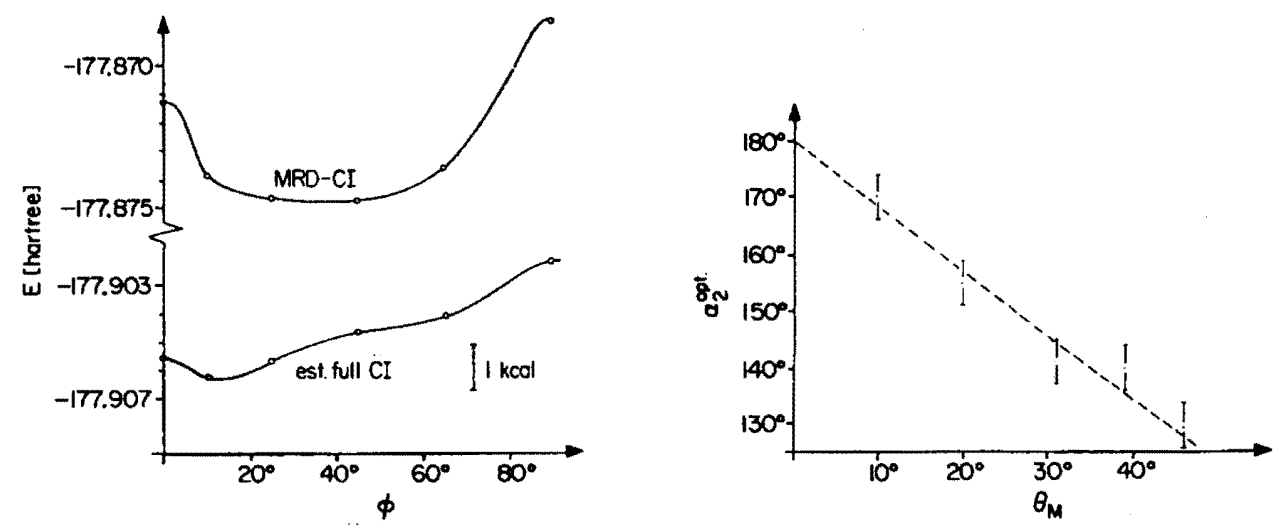

Fig. 5. Variation of the parameter $\Phi$ for the $\mathrm{FC}_{2} \mathrm{H}_{4}$ structure in which all other geometrical parameters are optimum.

Fig. 6. Calculated dependence of the optimum angle $\alpha_{2}$ on the variable $\theta_{M}$ in the calculations for $\mathrm{FC}_{2} \mathrm{H}_{4}$. 
motion is obtained by connecting the energy minima for each $\Theta_{M}$ value and is also indicated in Fig. 4.

In this connection it is interesting to examine the optimal values of $\alpha_{2}$ and $R_{\text {CC }}$ with angle $\theta_{M}$. An almost linear relationship between $\Theta_{M}$ and $\alpha_{2}$ is observed thereby as shown in Fig. 6; again this relation represents the change in $s p^{3}$ hybridization at the central carbon, $\mathrm{C2}$, from $\Theta_{M}=41^{\circ}$ to the $s p^{2}$ hybridization for $\theta_{M}=0$ in the symmetrical structure. Practically the same interdependence has been found for the other systems $\left(\mathrm{ClC}_{2} \mathrm{H}_{4}\right.$ and $\left.\mathrm{BrC}_{2} \mathrm{H}_{4}\right)$. In $\mathrm{ClC}_{2} \mathrm{H}_{4}$ additional calculations changing $\alpha_{1}$ and $\alpha_{2}$ between 150 and $210^{\circ}$ in the symmetric structure have also been carried out to test the influence of bending of the $\mathrm{CH}_{2}$ groups, but $\alpha=180^{\circ}$ has been found to be optimal; thus no further tests have been performed in $\mathrm{FC}_{2} \mathrm{H}_{4}$. The relationship between $\Theta_{M}$ and $R_{C c}$ seems to be somewhat less obvious, in particular since the magnitude of $\boldsymbol{R}_{M}$ also plays a role. This aspect has been studied in detail [17] but since the change in the total energy with this variable $R_{\mathrm{cc}}$ is relatively small it will not be discussed any further in the present context.

The following conclusions can be drawn from the information presented so far. (1) Because of the potential well in the energy surface around $\Theta_{M}=$ $0^{\circ}$, a shuttling motion would in principle be possible and would not necessarily compete directly with dissociation, whereby the barrier towards dissociation from the symmetrical structure is only about $4 \mathrm{kcal}$. (2) On the other hand, the energy difference between the minimum asymmetric structure and the optimal symmetric structure is about $33 \mathrm{kcal} \mathrm{mol}^{-1}$. Hence this barrier is so high that a shuttling motion for the fluorine is not expected. (3) The barrier to $\mathrm{CH}_{2}$ rotation is quite small $\left(<2 \mathrm{kcal} \mathrm{mol}^{-1}\right)$. In summary then, $\mathrm{FC}_{2} \mathrm{H}_{4}$ is expected to behave as a classical unbridged radical which appears to be entirely consistent with experimental evidence.

\section{THE SYSTEMS $\mathrm{BrC}_{2} \mathrm{H}_{4}$ AND $\mathrm{ClC}_{2} \mathrm{H}_{4}$}

The study of $\mathrm{BrC}_{2} \mathrm{H}_{4}$ was carried out in the same way as for $\mathrm{FC}_{2} \mathrm{H}_{4}$. While there are many similarities with respect to the interdependence of the various geometrical variables, the entire energetics in $\mathrm{BrC}_{2} \mathrm{H}_{4}$ is very different from that in the fluoro analogue. The calculated contour plots for the shuttling region are presented in Fig. 7.

First of all, the absolute minimum was found for an asymmetrical nuclear conformation. The optimal data (see Fig. 3) are: $\Theta=109^{\circ}, \mathrm{CC}=1.47 \AA$, $\alpha_{2}=135^{\circ}, \beta_{2}=110^{\circ}, \mathrm{C}-\mathrm{Br}=2.10 \AA$, whereby the values $\alpha_{1}=180^{\circ}$ and $\beta_{1}=118^{\circ}$ have been assumed. Compared to the corresponding fluorine data given before the difference between the values of $\alpha_{2}$ is not pertinent. The molecular angles $\mathrm{X}-\mathrm{C}-\mathrm{C}$ are essentially equivalent in both compounds. The CC distance seems to be somewhat smaller in $\mathrm{BrC}_{2} \mathrm{H}_{4}$ than in $\mathrm{FC}_{2} \mathrm{H}_{4}$ but the difference is small enough that an attempt at a qualitative explanation might be inadequate. The total energies are -2650.1875 hartree (SCF, electronic configuration $\left.1 a^{\prime 2} 2 a^{\prime 2} \ldots 18 a^{\prime 2} 19 a^{\prime} 1 a^{\prime \prime 2} \ldots 7 a^{\prime \prime 2}\right),-2650.497$ 


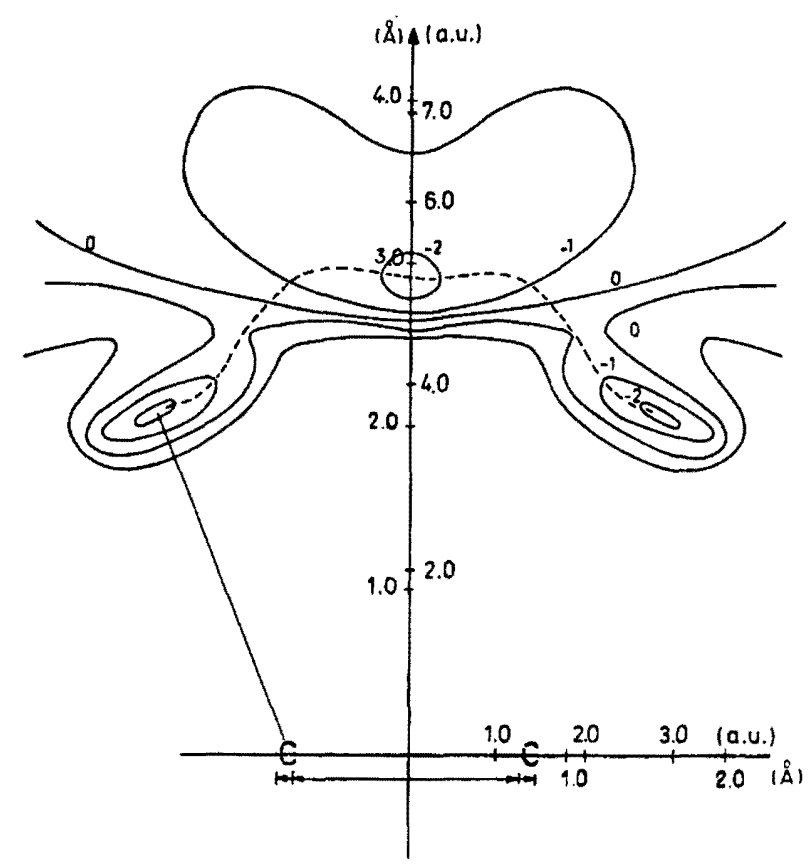

Fig. 7. Calculated contour plots for $\mathrm{BrC}_{2} \mathrm{H}_{4}$ in the shuttling region between the two carbons. All parameters are close to being optimized for each pair of $R_{M}, \theta_{M}$ values. The minimum energy path is also indicated. The contour lines correspond to 0,1 and $2 \mathrm{kcal} \mathrm{mol}^{-1}$ binding as indicated.

hartree (MRD-CI) and $\mathbf{- 2 6 5 0 . 5 2 2}$ hartree (full CI estimate). There is a second minimum in the path of symmetric approach by $\mathrm{Br}$ to $\mathrm{C}_{2} \mathrm{H}_{4}$ along $\Theta_{M}=0^{\circ}$, in the order of $2 \mathrm{kcal}$ below the dissociation limit.

The entire energy surface for a conceivable shuttling motion between the asymmetric minimum and the symmetrically bridged structure is extremely flat. While in $\mathrm{FC}_{2} \mathrm{H}_{4}$ the difference between equilibrium and the lowest symmetric point is $33 \mathrm{kcal}$, the corresponding values for $\mathrm{BrC}_{2} \mathrm{H}_{4}$ are only $1 \mathrm{kcal}$. If the minimum energy path is considered there seems to be a small barrier between the two minima corresponding to $\Theta_{M} 40^{\circ}$ and $\Theta_{M}=0^{\circ}$ (Fig. 8); it looks as if a 2 kcal activation is first necessary to get out of the equilibrium potential well corresponding to the asymmetric structure but that $1 \mathrm{kcal}$ is then gained in moving towards the symmetric bridge. Whether these differences are realistic or would change somewhat upon further geometry optimization can be disputed. Further calculations do not seem to be in order because of the small effect and in particular because the minimum energy path does not necessarily coincide with the reaction path since vibrational levels are also involved in the actual mechanism. On the other hand, a rigid analysis of the various calculations implies the presence of an absolute minimum in the total electronic energy corresponding to the unsymmetrical nuclear conformation. 

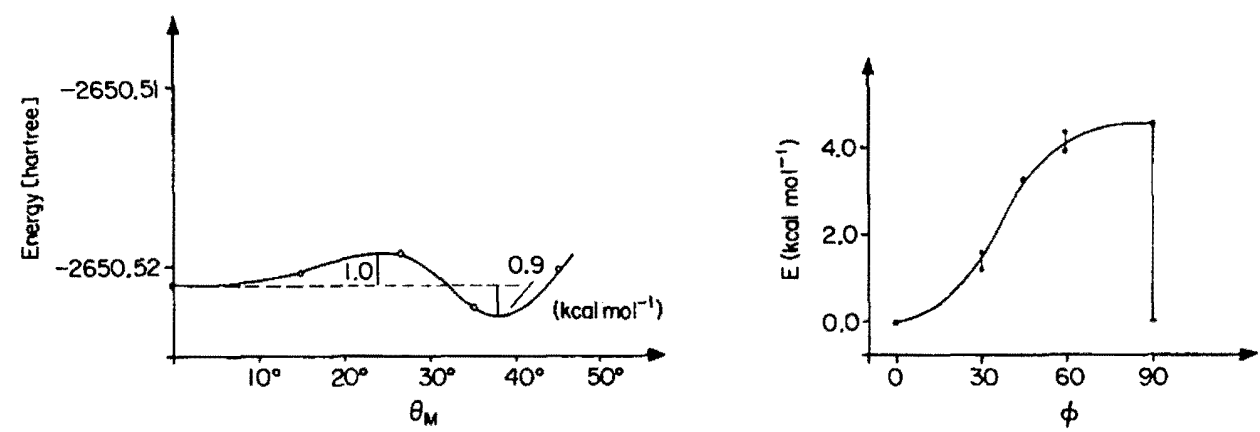

Fig. 8. Estimated energy path for the shuttling motion of $\mathrm{BrC}_{2} \mathrm{H}_{4}$.

Fig. 9. Calculated barrier to rotation (estimated full $\mathrm{Cl}$ values) for $\mathrm{ClC}_{2} \mathrm{H}_{4}$ at equilibrium.

Calculations on the $\mathrm{ClC}_{2} \mathrm{H}_{4}$ radical show a behavior between that of $\mathrm{FC}_{2} \mathrm{H}_{4}$ and $\mathrm{BrC}_{2} \mathrm{H}_{4}$. The absolute minimum is found for a $\beta$-chloroalkyl radical with a typical bond angle around $110^{\circ}$ and a CC bond separation close to a single bond. Again there seems to be a shallow secondminimum for the $\Theta_{M}=0$ arrangement whereby the difference in the two structures is in the order of $8 \mathrm{kcal} \mathrm{mol}^{-1}$. The MEP is currently under further investigation in order to see whether there is a barrier between the absolute minimum and the optimal $\Theta_{M}=0$ point, or whether a simple barrier separates the two asymmetrically bridged structures as has been found in the $\mathrm{FC}_{2} \mathrm{H}_{4} \mathrm{com}$ pound. In contrast to the SCF results obtained by Hopkinson et al. [18] the present calculations do not find the 2-chloroethyl radical to be pyramidal at the radical center, but as these authors [18] have already pointed out, inclusion of correlation energy, as in the present work, is required for quantitatively reliable results.

The barrier for $\mathrm{CH}_{2}$ rotation (i.e., the dependence of the energy on the angle $\Phi$ ) has also been investigated in the bromo and chloro compounds. CI calculations find a barrier of about $4 \mathrm{kcal} \mathrm{mol}^{-1}$ for $\mathrm{ClC}_{2} \mathrm{H}_{4}$ ( $3 \mathrm{kcal}$ in SCF calculations) as shown in Fig. 9. This computation has been carried out without the use of $d$-functions on carbon (because the size of the problem has increased considerably in this case in which no symmetry element is present any more) but it is expected that the magnitude would remain the same even after the addition of such functions. The $\mathrm{Cl}$ was performed by using a 2-root and 1-root configuration selection as well as different MO basis sets; the corresponding spread in values is indicated in the Figure by vertical bars. It is seen that the minimum is definitely for $\Phi=0^{\circ}$, unlike the situation in the fluoro radical. The equivalent calculation has been undertaken for $\mathrm{BrC}_{2} \mathrm{H}_{4}$ and the calculated energy barrier was found to be ca. 4-5 $\mathrm{kcal} \mathrm{mol}^{-1}$ (depending on the full $\mathrm{CI}$ and MRD-CI value).

In summary then, in the bromo-alkyl radical the energy minimum is found at the asymmetric nuclear conformation, but the calculated potential surface suggests that shuttling is energetically very favorable and should 
be the preferred process compared to $\mathrm{CH}_{2}$ rotation. The $\mathrm{ClC}_{2} \mathrm{H}_{4}$ radical seems to be closer in its behavior to $\mathrm{BrC}_{2} \mathrm{H}_{4}$ and more detailed quantitative data are in preparation. Finally, it should be stressed that large-scale CI calculations (together with an appropriate AO basis including $d$ functions) are essential in this study since the SCF treatment along gives much less realistic dissociation data and generates only repulsive curves for the symmetric $\left(\Theta_{M}=0\right)$ approach of the halogen. More details of the present work, in particular its relation to experimental evidence, will appear elsewhere [7].

\section{REFERENCES}

1 (a) P. S. Skell and J. G. Traynham, Acc. Chem. Res, A, (1984) 60.

(b) W. J. Thaler, J. Am. Chem. Soc., 85 (1963) 2607.

2 A good summary of the experimental results is contained in: J. K. Kochi, Free Radicals, Vol. II, Wiley-Interscience, New York, Chap. 26, 1973.

3 See for example, P. S. Skell and J. G. Traynham, Acc. Chem. Res., 17 (1984) 160 and references therein.

4 See for example: D. D. Tanner, D. Darwish, M. Mosher and N. J. Bunce, J. Am. Chem. Soc., 91 (1969) 7398. D. D. Tanner, W. M. Mosher, N. C. Das and E. V. Blackburn, J. Am. Chem. Soc., 93 (1971) 4802 and further refs. in [2] and [3].

5 P. J. Krusic and J. K. Kochi, J. Am. Chem. Soc., 93 (1971) 846.

6 L. Lunazzi, G. Placucci, L. Grossi and M. Guerra, J. Chem. Soc., Perkin Trans., 43 (1982) 2.

7 B. Engels, S. D. Peyerimhoff and P. S. Skell, in preparation.

8 (a) T. H. Dunning, J. Chem. Phys., 53 (1970) 53.

(b) T. H. Dunning and J. P. Hay, in H. F. Schaefer (Ed.), Modern Theoretical Chemistry, Plenum Press, New York, Vol. 3, 1976, p. 1.

10 T. H. Dunning, J. Chem. Phys., 66 (1977) 1382.

11 R. J. Buenker and S. D. Peyerimhoff, in P. O. Löwdin and B. Pullmann (Eds.), New Horizons of Quantum Chemistry, D. Reidel, Dordrecht, 1983, p. 183.

12 R. S. Mulliken, Rev. Mod. Phys., 14 (1942) 204.

13 A. D. Walsh, J. Chem. Soc., (1953) 2260 and following articles.

14 R. J. Buenker and S. D. Peyerimhoff, Chem. Rev., 74 (1974) 127.

15 S. K. Shih, R. J. Buenker, S. D. Peyerimhoff and C. J. Michejda, J. Am. Chem. Soc., 94 (1972) 7620.

16 D. J. Edge and J. K. Kochi, J. Am. Chem. Soc., 94 (1972) 6485.

17 B. Engels, Diplomarbeit, Bonn, 1985.

18 A. C. Hopkinson, M. H. Lien and I. G. Csizmadia, Chem. Phys. Lett., 71 (1980) 557. 